\title{
Is the Salvation of Life on the Planet?
}

\section{Halidullin 0*}

Academician of the Russian Federation LAN, Kazakh National University, Russia

*Corresponding author: Oleg Halidullin, Academician of the Russian Federation LAN, Kazakh National University, Russia, Tel: +87770550099; Email: 7115215@mail.ru

\section{Opinion \\ Volume 1 Issue 1}

Received Date: August 25, 2018

Published Date: September 19, 2018

DOI: $10.23880 /$ oajwx-16000106

\section{Opinion}

Before the advent of modern industry, nature had tangible additions in the composition of atmospheric water vapor - natural evaporation from transpiration of plant and natural emissions, in particular, from the respiration of creatures, animal worlds. Every living being, every plant in its secretions, at the molecular level, in its secretions creates information about itself. It is known that in the exhalation of a living being in the moisture of breathing, in any secretions, information is contained. It is proved by the fact that animals determine their victims and the opposite sex by smells. Plants allocate their personal phytoncides. Phytoncides are all volatile substances released by plants, including those that are practically impossible to collect in appreciable amounts. These phytoncides are also called "native antimicrobial substances of plants"http://stgetman.narod.ru/fitoncid.html. We all enjoy the smells of flowers, coniferous forests, shrubs. Medicine has such achievements that it is possible to diagnose his illnesses according to the exhalation of a person. https://www.golos-ameriki.ru/a/254391.html: The diagnosis of exhalation is the latest achievements of medicine, a revolution in oncology.

Inspectors of road safety services for smell movement and appliances determine the state of alcohol status of the driver of the vehicle. All these smells and volatile substances are formed inside the living organism as a result of the most complicated chemical transformations. We also know that water has a different molecular structure and carries information. Maybe this information is preserved in evaporation? For example, in the works of the famous Japanese scientist Emoto Mazaru, it is clearly defined how ice crystals change depending on sound and even mental effects on water. Proceeding from this, it can be concluded that evaporation from organic matter also has certain properties and, accumulating in the atmosphere, creates certain conditions. It is these conditions that form the volumes of subsequent precipitation, the location of their distribution, and the graphs of precipitation.

A man with his civilization influences the path of organic transformations, or rather destroys a whole link in the everlasting path of water-food chains. This is asphalt, arable land, surface water bodies, and landfills. According to the area of the entire land area of the planet, such territories became 63 percent for 2015, which are expanding with the development of technologies with increasing acceleration. Even greater evaporation produces man from production and communal industries. Water coming from the water pipe evaporates in millions of evaporators, heaters, sinks, dryers from each rag, from each washed cup without changing the structure in the organics. These are evaporation unknown to the nature, let's call them artificial.

Nothing in nature is done just like that. Nothing never disappears anywhere and does not appear anywhere. What is the further path of artificial fumes? What is their structure, what their volume is and what is the speed of such evaporation. We feel it ourselves. They rise in the sky in huge volumes, they permanently cover the sun. They fall out incredible precipitation in some places, and do not carry drops into others. The mechanism of rational circulation has disappeared. This is no longer a cycle of water in nature, but a cycle of artificial fumes. Nature is powerless to control such volumes and such velocities.

It follows that organic evaporation, uniting in the clouds, synthesizes a certain substance, some special property that somehow forms the temperature, volume, 
shape, quality of the surface atmosphere. Interacting with the biota for millions of years, a certain cycle of substances between the atmosphere and the soil has stabilized. The quality, the amount of precipitation, the places of their condensation, movement in geographic space and loss - all this was polished in a single process. This explains the creation and distribution of all natural areas from deserts to the tropics.

From here we get droughts in some areas, floods in others. Destroyed, created by millions of years, the mechanism of atmospheric phenomena. In effect, the destruction of just one link in the circuit of water on the planet led to the creation of an entirely new atmospheric mechanism - the circulation of artificial fumes, a new source of natural disasters, to climate change.

\section{Is there a Possibility of Salvation?}

The Paris Agreements of 2015, "green technologies", alternative energy sources - this is an intuitive direction created by mankind to conserve nature right direction.
But only these are microscopic steps. Moreover, these steps lead to a catastrophe, diverting the efforts of mankind to preserve life.

The only true direction to preserving life on the planet is the movement to reduce artificial and return natural vapors. If we want to preserve life on the planet, we must begin to restore the nature of the cycle of water selected from it to nature.

A new concept for stopping climate change should be developed. The main elements of this are

- Complete and unconditional stopping of projects and construction of all types of works with the partitioning of rivers, the laying of new channels

- Global water saving in everyday life and production of all products by every person, every production of all countries and continents

- Maximum possible rapid relocation of all types of production, the infrastructure of cities and the very housing under the ground. 